\title{
Synthesis of poly(furfuryl alcohol)/montmorillonite nanocomposites by direct in-situ polymerization
}

\author{
DJAMAL EDDINE KHERROUB ${ }^{1, *}$, MOHAMMED BELBACHIR ${ }^{\mathbf{1}}$ and SAAD LAMOURI ${ }^{\mathbf{2}}$ \\ ${ }^{1}$ Laboratory of Polymer Chemistry, Department of Chemistry, Faculty of Sciences, University of Oran, BP 1524, \\ El'Menouer, Oran 31000, Algeria \\ ${ }^{2}$ Laboratory of Macromolecular Chemistry, Polytechnic Military School (EMP), Bordj El Bahri, 16111 Algiers, Algeria
}

MS received 18 January 2014; revised 19 March 2014

\begin{abstract}
The purpose of this study was to obtain poly(furfuryl alcohol) nanocomposites with Algerian organically modified clay (termed 12-montmorillonite). The formation of poly(furfuryl alcohol) was confirmed by infrared spectroscopy (IR); the prepared nanocomposites were characterized by X-ray diffraction (XRD), transmission electron microscopy (TEM) and thermogravimetric analysis (TGA). The evolution of mechanical properties was also studied. The obtained results confirm the intercalation of molecules of salt in the clay layers, and a good interaction with the polymer, showing the formation of intercalated and/or exfoliated structures. The nanocomposites showed higher thermal stability compared to pure polymer, and the mechanical properties presented interesting and promising results.
\end{abstract}

Keywords. 12-Montmorillonite; nanocomposites; poly(furfuryl alcohol); exfoliation.

\section{Introduction}

During the last decade, polymer/clay nanocomposites have received increasing attention from scientists and industrial researchers because they generally exhibit greatly improved mechanical, thermal, barrier and flame-retardant properties at low clay content in comparison with unfilled polymers or more conventional microcomposites. ${ }^{1-10}$

Poly(furfuryl alcohol) (PFA) is a biopolymer which is of considerable interest due to its commercial availability, low viscosity, which lends it to applications involving in-situ polymerization, and finally, its compatibility with hydrophilic nanoparticles. Surprisingly, PFA has received little attention to date, compared to other biopolymers. While PFA is hydrophobic, its monomer precursor furfuryl alcohol (FA) is hydrophilic and completely soluble in water due to the hydroxyl group of the side chain and the oxygen heteroatom of the furan ring. The presence of these functional groups in FA makes this monomer able to stabilize suspensions of hydrophilic nanoparticles such as, for example, the hydroxylated surfaces of montmorillonite (MMT). ${ }^{11}$ FA has been polymerized by several organic, mineral and Lewis acids. ${ }^{12-17}$ The acid-catalyzed polymerization of FA inevitably leads to a black, crosslinked product. There is no doubt that the first phase of this complex process consists of condensation reactions between the $\mathrm{OH}$ group on one hand and either an $\mathrm{H} 5$ atom of a furan ring (head-to-tail

\footnotetext{
*Author for correspondence (djamaleddine.kherroub@yahoo.com)
}

structure, 1) or another $\mathrm{OH}$ group (head-to-head structure, 2), on the other. This series of acid-catalysed condensation steps produces linear oligomers which therefore can contain both types of bridges, namely methylene (1) and dimethyleneoxide (2) moieties. It has been established that, under strongly acidic conditions, the latter structure has a tendency to lose formaldehyde and revert to the former, as indicated in the simplified scheme (scheme 1). This phenomenon, coupled with the higher kinetic propensity for head-to-tail condensation, results in macromolecular structures in which methylene bridges are in general much more frequent than their other counterparts. Many studies have been carried out in order to clarify the mechanism of coloration and crosslinking. It was concluded that hydrogen atoms of the methylene bridges between two furan rings play essential role in favouring the side reactions related to colour formation and crosslinking of chains. ${ }^{13-15,18-20}$

In this work we use the MMT to synthesize PFA nanocomposites, which serve multiple functions, by first catalysing the polymerization of FA, and then acting as an effective matrix modifier. The most frequently employed layered silicates, such as MMT, are of smectite type and belong to the family of 2:1 phyllosilicates. Their crystalline structure is composed of stacked layers made of two silica tetrahedrons fused to an edge-shared octahedral sheet of aluminium or magnesium hydroxide. Pristine clays are hydrophilic and thus immiscible with most polymers, due to alkali cations residing in the interlayer to counterbalance the negative charge generated by isomorphic substitutions within the silicate sheets. However, the polymer-clay compatibility can be improved via ion-exchange reactions 


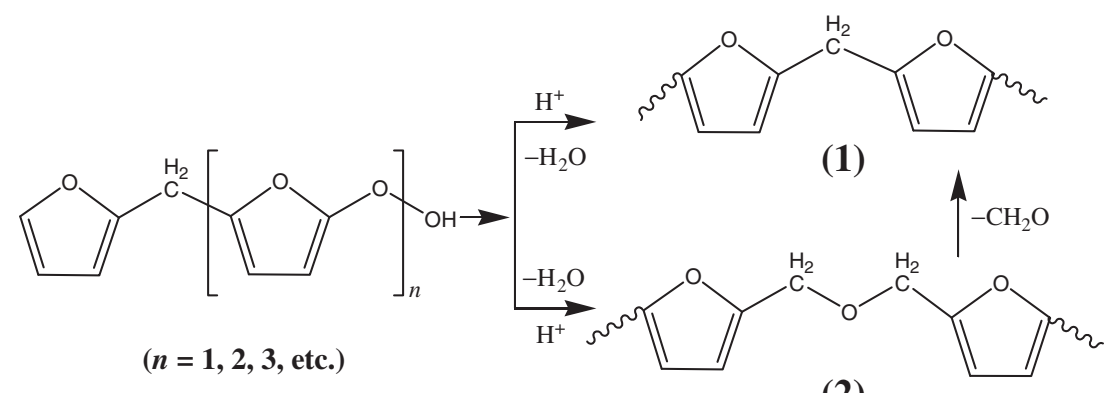

(2)

Scheme 1. Polymerization of furfuryl alcohol.

with organic surfactants, including mainly alkylammonium cations. ${ }^{10,21}$

Therefore, the purpose of this study was to obtain PFA nanocomposites with national organically modified clay. The novelty of this work is the fact that we use clay of our region, which constitutes low costs and has excellent properties.

\section{Experimental}

\subsection{Materials}

FA (grade 99\%) and 12-aminolauric acid were used as purchased from Aldrich Chemical. Raw-Maghnite: Algerian MMT clay which has been used as catalyst is supplied by a local company (ENOF Maghnia (Western of Algeria)). Its chemical composition is given in table 1 .

\subsection{Methodology}

2.2a Preparation of Maghnite-Na: Maghnite-Na was prepared according to the process reported in our previous study. ${ }^{22,23}$ Raw-Maghnite $(20 \mathrm{~g})$ was crushed for $20 \mathrm{~min}$ using a prolabo ceramic balls grinder. It was then dried for $2 \mathrm{~h}$ at $105^{\circ} \mathrm{C}$. The Maghnite was placed in an Erlenmeyer flask together with $500 \mathrm{ml}$ of $1 \mathrm{M} \mathrm{NaCl}$. The Maghnite/water mixture was stirred using a magnetic stirrer until saturation was achieved after $3 \mathrm{~h}$ at room temperature; the mineral was then washed with distilled water to make it $\mathrm{Cl}^{-}$free and then dried at $105^{\circ} \mathrm{C}$.

2.2b Preparation of 12-MMT: Natural Na-MMT is hydrophylic and not compatible with most organic molecules. The sodium cation in the interlayer space of MMT can be exchanged with organic cations to yield organophilic MMT. For the present purpose, polymerization in the interlayer space, ammonium cations of $\omega$-amino acid were chosen as cations since their $-\mathrm{COOH}$ group catalyses the polymerization of FA. In a $1000 \mathrm{ml}$ beaker, $24 \mathrm{mmol}$ of 12-aminolauric acid, $2.4 \mathrm{ml}$ of concentrated hydrochloric acid and $200 \mathrm{ml}$ of water were added at $80^{\circ} \mathrm{C}$. The solution of the 12-aminolauric acid was added to a dispersion composed of $10 \mathrm{~g}$ of MMT-Na and $1000 \mathrm{ml}$ of hot water, and
Table 1. Chemical composition of the catalyst.

\begin{tabular}{lc}
\hline Species & $\%(w / w)$ \\
\hline $\mathrm{SiO}_{2}$ & 69.4 \\
$\mathrm{Al}_{2} \mathrm{O}_{3}$ & 14.7 \\
$\mathrm{Fe}_{2} \mathrm{O}_{3}$ & 1.2 \\
$\mathrm{MgO}$ & 1.1 \\
$\mathrm{CaO}$ & 0.3 \\
$\mathrm{Na}_{2} \mathrm{O}$ & 0.5 \\
$\mathrm{~K}_{2} \mathrm{O}$ & 0.8 \\
$\mathrm{TiO}_{2}$ & 0.2 \\
$\mathrm{As}$ & 0.05 \\
L.O.I. & 11 \\
\hline
\end{tabular}

L.O.I.: Loss on ignition at $900^{\circ} \mathrm{C}$ in wt $\%$.

then this mixture was stirred vigorously for $10 \mathrm{~min}$, giving a white precipitate. The product was filtered, washed with hot water, and freeze-dried. In this paper, we call the cation exchanged MMTs '12-montmorillonite', where $n$ is the carbon number of the 12-aminolauric acid. ${ }^{24}$ The basal spacings (interlayer distance) of the samples were obtained from the peak position of the X-ray diffraction (XRD) pattern.

2.2c Preparation of the nanocomposites: In a roundbottom flask, $10 \mathrm{~g}$ (102 mmol) of FA and 1, 3, 5, 7, 10 and $15 \mathrm{wt} \%$ of 12-MMT were placed. For all six experiments, the mixture was gradually heated to $120^{\circ} \mathrm{C}$; at this temperature the reaction was allowed for $2 \mathrm{~h}$, yielding a polymeric product. During the reaction, the homogenization of the reaction medium is carried out by vigorous stirring with a magnetic stir bar. ${ }^{11}$ After cooling, the product was mechanically crushed. The fine particles were washed with 2:1 of water at $80^{\circ} \mathrm{C}$ for $1 \mathrm{~h}$; the nanocomposites will then be denoted as PFANC1, PFANC3, PFANC5, PAFNC7, PFANC10 and PFANC15 according to their amount of clay.

\subsection{Characterization}

2.3a Infrared spectroscopy (IR): IR analyses were carried out using the Perkin Elmer Spectrum Two FT-IR with UATR sampling accessory. 
2.3b X-ray diffraction (XRD): XRD analysis was carried out at room temperature on a Bruker D8 Advance X-Ray diffractometer $(40 \mathrm{kV}, 30 \mathrm{~mA})$ using $\mathrm{CuK} \alpha$ radiation $(\lambda=$ $0.154 \mathrm{~nm})$ at the rate of $2^{\circ} \mathrm{min}^{-1}$ in the $2 \theta$ range of $2.0-80^{\circ}$.

2.3c Transmission electron microscope (TEM): The phase morphologies of the nanocomposites were observed by a TEM, Philips CM 120, operating at an acceleration voltage of $120 \mathrm{kV}$. Samples were cryogenically microtomed $\left(-70^{\circ} \mathrm{C}\right)$ into ultrathin sections $(30 \mathrm{~nm}$ thick) from films with a diamond knife using an RMC MT-7000.

2.3d Thermogravimetry (TG): The TG technique was also used, by means of a TGA 51 Shimadzu equipment, operating at a heating rate of $10.0^{\circ} \mathrm{C} \mathrm{min}^{-1}$, from room

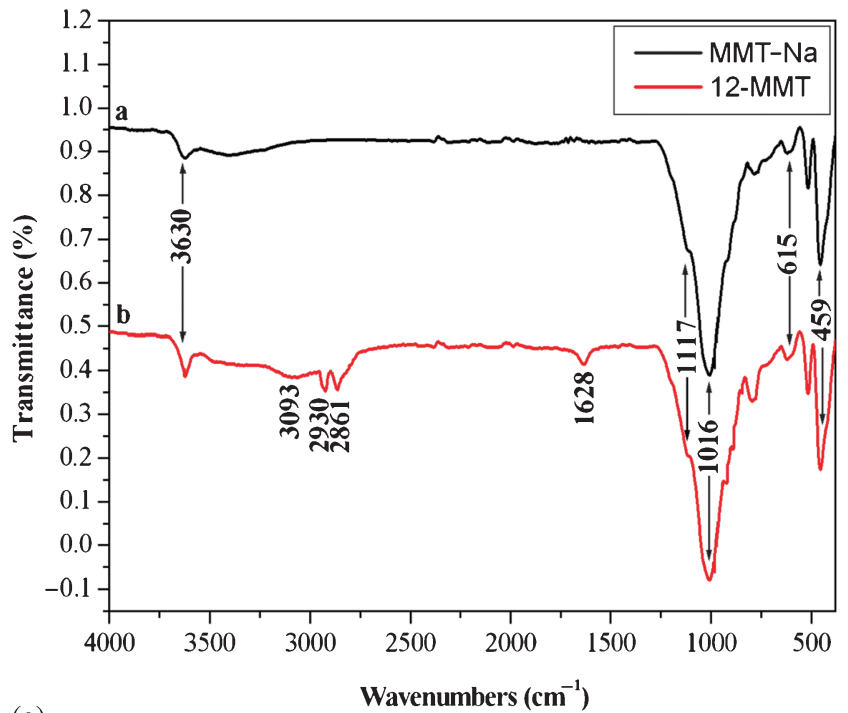

(a)

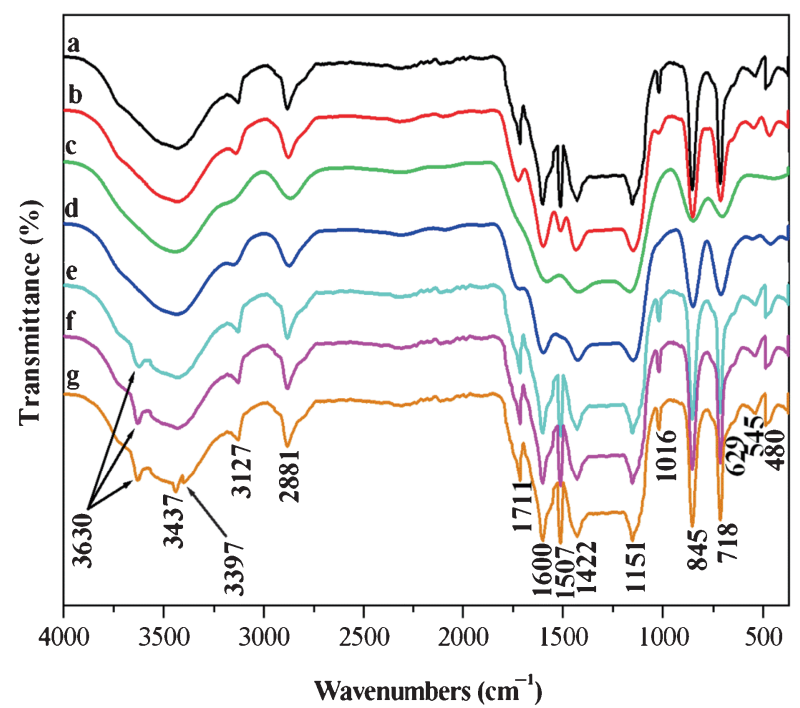

(b)

Figure 1. (a) FT-IR spectra for (a) MMT-Na and (b) 12-MMT. (b) FT-IR spectra for (a) virgin PFA; (b) PFANC1; (c) PFANC3; (d) PFANC5; (e) PFANC7; (f) PFANC10 and (g) PFANC15. temperature up to $900^{\circ} \mathrm{C}$ under air atmosphere with a flow rate of $50 \mathrm{ml} \mathrm{min}^{-1}$. The sample mass was $5.0 \pm 0.5 \mathrm{mg}$. The TG curves were analyzed with the aid of the TASYS software from Shimadzu.

2.3e Mechanical properties: Tensile tests were conducted using a LLoyd LR/10KN Universal Machine at room temperature and crosshead speed of $50 \mathrm{~mm} \mathrm{~min}^{-1}$ for the determination of tensile modulus and yield strength, according to the standard ASTM D638.

\section{Results and discussion}

\subsection{Infrared spectroscopy}

In figure 1a, the IR data of MMT-Na and 12-MMT are given. The intensity of the absorption band at $3630 \mathrm{~cm}^{-1}$

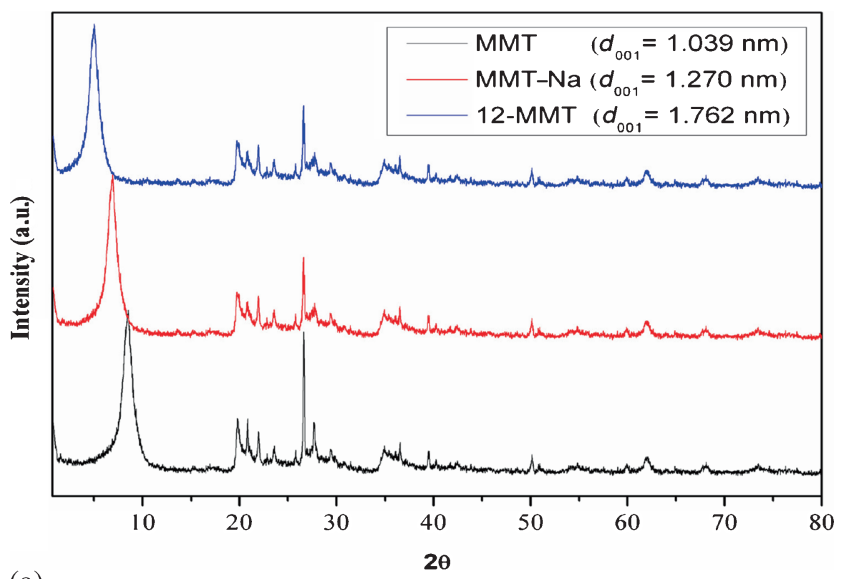

(a)

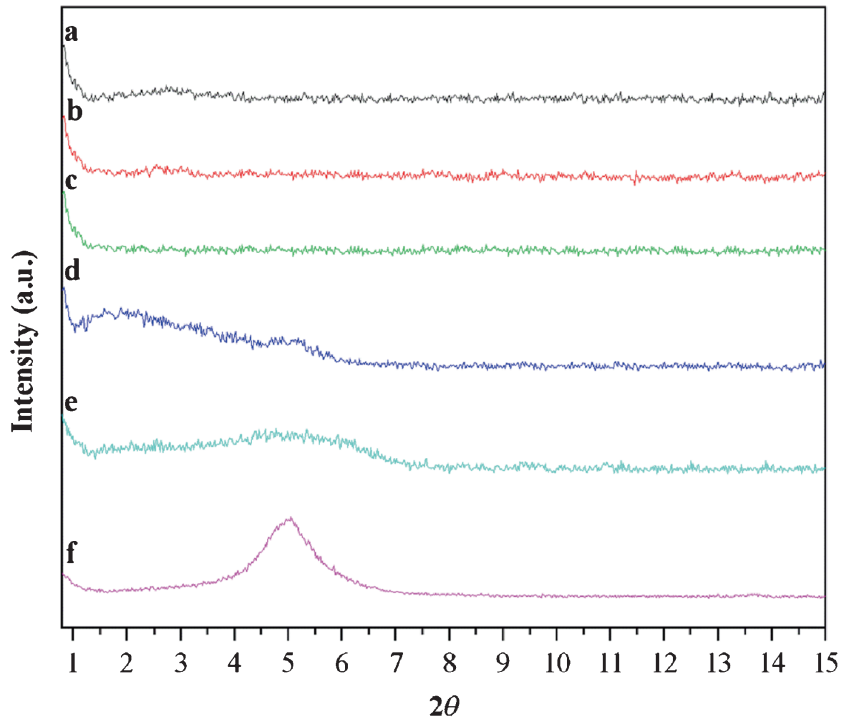

(b)

Figure 2. (a) XRD patterns of the clay without treatment (MMT), clay treated with $\mathrm{NaCl}$ (MMT-Na), and organophilic clay (12-MMT). (b) XRD patterns for (a) PFANC1; (b) PFANC3; (c) PFANC5; (d) PFANC7; (e) PFANC10 and (f) PFANC15. 
is attributed to $\mathrm{AlAlOH}$ coupled by $\mathrm{AlMgOH}$ stretching vibrations. The $\mathrm{Si}-\mathrm{O}$ out of plane and $\mathrm{Si}-\mathrm{O}-\mathrm{Si}$ in plane stretching bands are shown at 1117 and $1016 \mathrm{~cm}^{-1}$, respectively. The signal at $615 \mathrm{~cm}^{-1}$ is due to either $\mathrm{Al}-\mathrm{OH}$ or $\mathrm{Si}-\mathrm{O}$ bending and/or $\mathrm{Al}-\mathrm{O}$ stretching vibration. The band at $459 \mathrm{~cm}^{-1}$ is assigned to the $\mathrm{Si}-\mathrm{O}-\mathrm{Al}$ and $\mathrm{Si}-\mathrm{O}-\mathrm{Mg}$ coupled to $\mathrm{OH}$ vibration or $\mathrm{Si}-\mathrm{O}$ bending vibrations. ${ }^{25}$ Further from figure 1a, the IR spectrum of 12-MMT shows features that are a combination of characteristic bands of MMT and 12-aminolauric acid. The broad band in the region of $3250-3030 \mathrm{~cm}^{-1}$ is assigned to ionic bonded $\mathrm{N}-\mathrm{H}$ stretching band. The new bands at 2930 and $2861 \mathrm{~cm}^{-1}$ are, respectively, attributed to $\mathrm{C}=\mathrm{H}$ asymmetric/symmetric stretching. The combination of $\mathrm{O}-\mathrm{H}$ deformation and $\mathrm{N}-\mathrm{H}$ stretching is assigned to $1628 \mathrm{~cm}^{-1} .{ }^{26}$ The results obtained by IR analyses show clearly the functionalization of the clay.

The IR measurements of virgin PFA and PFANCs are in a good agreement with PFA structure (figure 1b), and they do not exhibit significant differences respect to those obtained with several researchers. ${ }^{12-15,27}$ All IR spectrums show the presence of signals at 718, 1016, 1151, 1507 and $3127 \mathrm{~cm}^{-1}$ that are attributed to furan rings. Signals appearing at 845 and $1600 \mathrm{~cm}^{-1}$ are, respectively, due to bending out of the plane of the $\mathrm{CH}$ linkage and to the stretching of the $-\mathrm{C}=\mathrm{C}-$ groups in the $2-5$ disubstituted furanic rings. Aliphatic segments give rise to the bands at 2881 and $1422 \mathrm{~cm}^{-1}$, whereas the signal at $3437 \mathrm{~cm}^{-1}$ is assigned to the stretching of the $\mathrm{OH}$ end groups in the PFA. Due to the opening of some furan rings, carbonylic structures are formed and characterized by absorption band at $1711 \mathrm{~cm}^{-1}$. The weak signal at $629 \mathrm{~cm}^{-1}$ is assigned to the coupling of $\mathrm{Si}-\mathrm{O}$ and $\mathrm{Al}-\mathrm{O}$ out of plane. ${ }^{26,28}$ The signals appearing at 545 and $480 \mathrm{~cm}^{-1}$ are, respectively, due to the $\mathrm{Mg}-\mathrm{OH}$ and $\mathrm{Al}-\mathrm{OH}$ groups. ${ }^{29}$ The appearance of the absorption band at $3630 \mathrm{~cm}^{-1}$ for PFANC7, PFANC10 and PFANC15 corresponds to $\mathrm{AlAlOH}$ coupled by $\mathrm{AlMgOH}$ stretching vibrations in the MMT; the absorption band at $3397 \mathrm{~cm}^{-1}$ in PFANC15 may be explained by absorption of interlayer water by montmorillonite. ${ }^{30}$
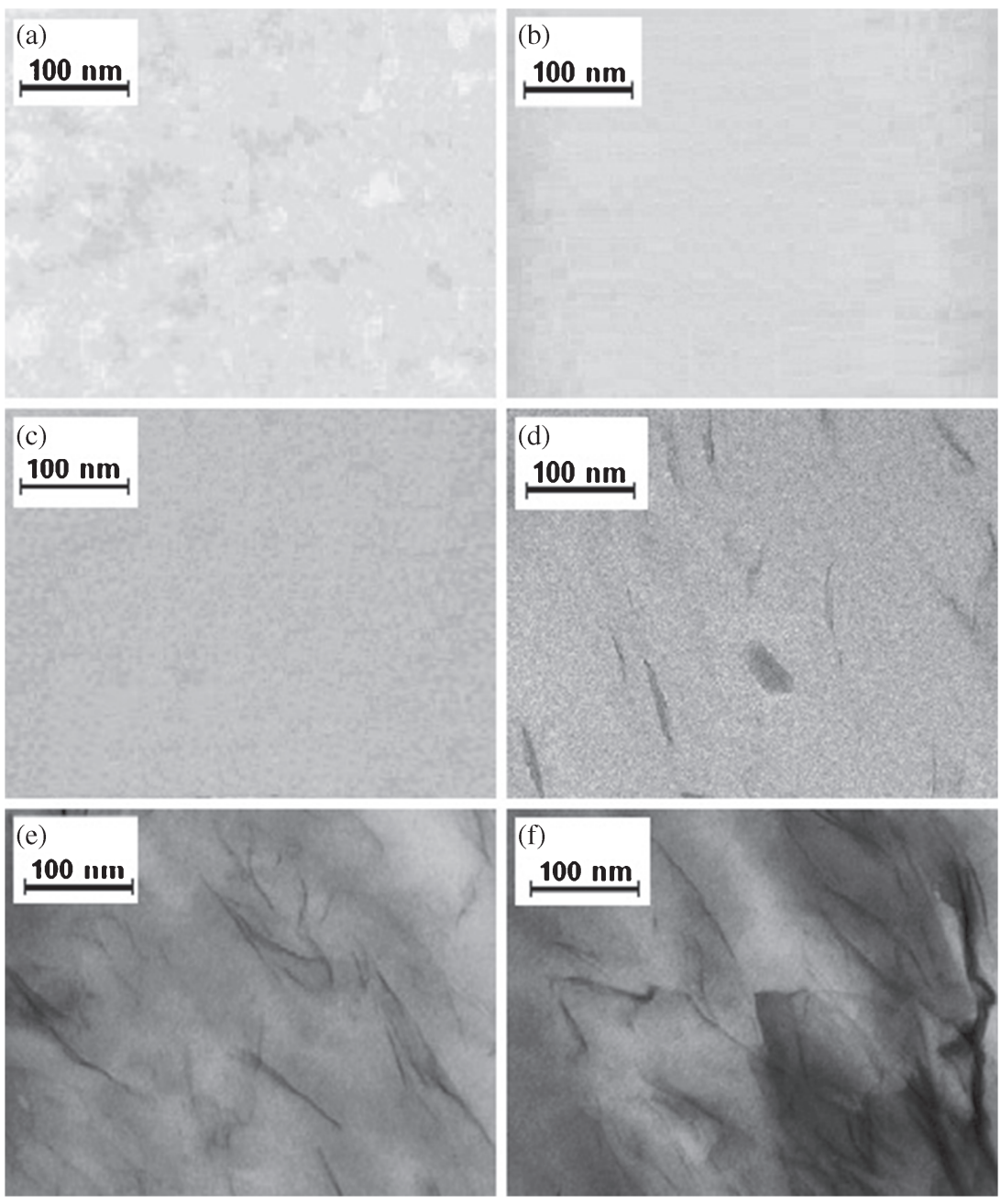

Figure 3. TEM images of: (a) PFANC1, (b) PFANC3, (c) PFANC5, (d) PFANC7, (e) PFANC10 and (f) PFANC15. 


\section{$3.2 X$-ray diffraction $(X R D)$}

The basal spacings (interlayer distance) of the raw clay (MMT), clay treated with $\mathrm{NaCl}$ (MMT-Na), organophilic MMT (12-MMT) and their nanocomposites with PFA were obtained from the peak position of the XRD pattern. The MMT shows the characteristic peak of the MMT at $2 \theta=$ 8.50 , corresponding to the interlayer distance ( $d_{001}$-spacing) of $10.39 \AA$. For the MMT-Na, the peak of the MMT is found

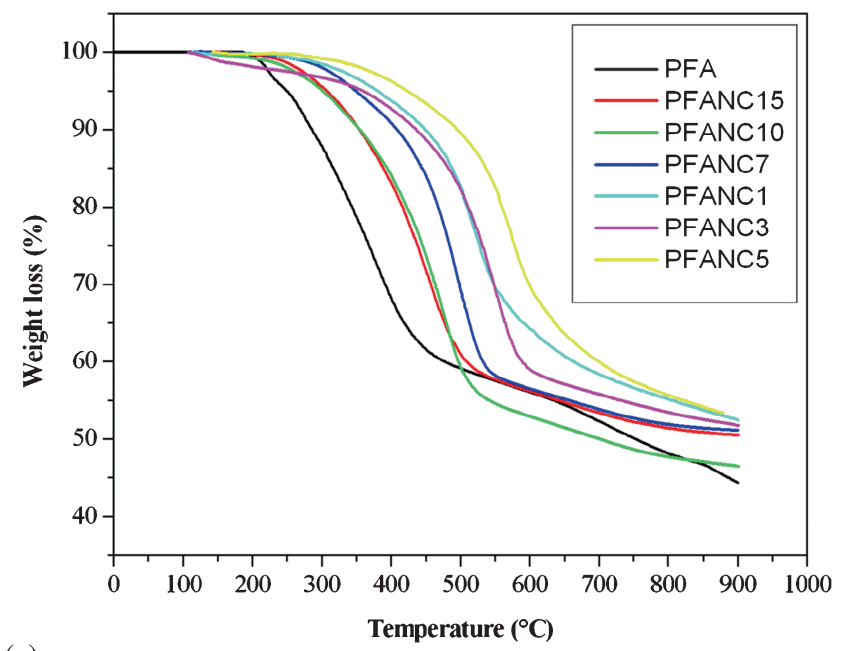

(a)

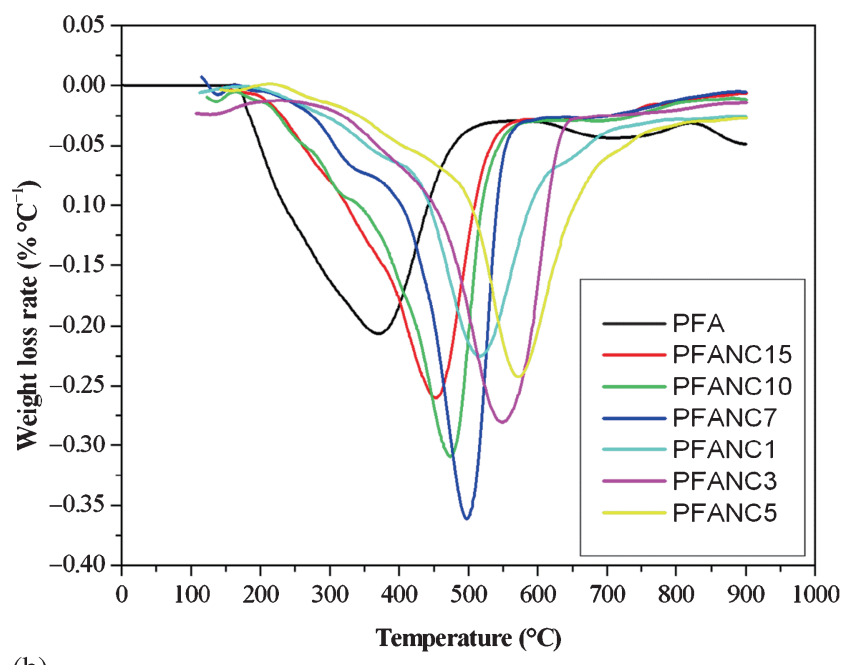

(b)

Figure 4. (a) TG curves of poly(furfuryl alcohol) and its nanocomposites. (b) DTG curves of poly(furfuryl alcohol) and its nanocomposites. at $2 \theta=6.95$, corresponding to the interlayer distance $\left(d_{001}\right.$ spacing) of $12.70 \AA$. The $d_{001}$ peak for the 12-MMT clay was shifted to a lower region $(2 \theta=5.01)$, indicating the increase in $d_{001}$-spacing ( $=17.62 \AA$ ) in the modified clay. An increase in $d_{001}$-spacing for $17.6 \AA$ was previously found by KS Katti et $a l^{26}$ when the organic moiety replaced the intra-gallery $\mathrm{Na}^{+}$of MMT-Na, resulting in 12-MMT. ${ }^{31}$ Our result unambiguously led us to assume the replacement of smaller $\mathrm{Na}^{+}$ ions by bulky molecules of 12-aminolauric acid inside the clay galleries that resulted in the increase in the gallery height of the MMT layers during ion-exchange reaction (figure 2a).

Figure $2 \mathrm{~b}$ shows the XRD analysis of different nanocomposites prepared $(1,3,5,7,10$ and $15 \mathrm{wt} \%)$. The strong diffraction peak of the pristine clay (12-MMT) is not observed on the patterns of PFANC1, PFANC3 and PFANC5; the clays are rather well exfoliated in the PFA matrix at low filler contents, although we can observe a bump for PFANC1 and PFANC3; this indicates that there is also a partial exfoliation. As the clay content increases, i.e., for PFANC7, PFANC10 and PFANC15, a diffraction peak at $2 \theta=4.86,4.94$ and 4.95 , respectively, is seen; this is the peak of MMT which confirms the existence of an immiscible phase. Moreover, another peak is observed at $2 \theta=2.02$ and 2.32 for PFANC7 and PFANC10, respectively, in which clay layers are not exfoliated whereas they are intercalated by the polymer chains as suggested by the increase of the $d_{001}$ value.

\subsection{Transmission electron microscope (TEM)}

The TEM analysis was further used to study in detail the filler dispersion state. The obtained images through TEM analysis let us presume that the fillers were homogeneously dispersed in the PFA matrix for the low rates (PFANC1, PFANC3 and PFANC5); the inorganic layers are parallel to the film surface and they are mainly well dispersed. On the other hand the intercalated structure of nanocomposites PFA/12-MMT for high clay content is fully confirmed (PFANC7, PFANC10 and PFANC15), with the existence of intercalated/immiscible or immiscible phases (intense regions), especially for PFANC15. Figure 3 shows that, with 1, 3 and $5 \mathrm{wt} \%$ 12-MMT, individual clay platelets are randomly distributed in the PFA matrix and confirm the exfoliated structure, and also intercalated, immiscible and intercalated/immiscible structures for 7, 10 and $15 \mathrm{wt} \%$ clay content. The morphological description obtained by TEM support the results obtained by XRD analysis.

Table 2. TGA results of PFA and nanocomposites.

\begin{tabular}{lrrrrrrr}
\hline & & \multicolumn{6}{c}{ Nanocomposites with various clay content (wt\%) } \\
\cline { 3 - 8 } & \multicolumn{1}{c}{ PFA } & \multicolumn{1}{c}{1} & \multicolumn{1}{c}{3} & \multicolumn{1}{c}{5} & \multicolumn{1}{c}{7} & \multicolumn{1}{c}{10} & \multicolumn{1}{c}{15} \\
\hline$T_{10 \%}$ & 307.24 & 435.92 & 447.97 & 494.32 & 408.55 & 354.06 & 353.72 \\
$T_{60 \%}$ & 445.47 & 589.10 & 661.50 & 698.39 & 532.12 & 496.93 & 508.60 \\
Residue (wt\%) & 41.20 & 51.77 & 52.45 & 53.34 & 51.12 & 46.49 & 50.51 \\
\hline
\end{tabular}


Table 3. Mechanical properties of PFA and its nanocomposites.

\begin{tabular}{lcc}
\hline Specimens & Tensile modulus $(\mathrm{GPa})$ & Yield strength $(\mathrm{MPa})$ \\
\hline PFA & 0.81 & 09 \\
PFANC1 & 1.03 & 15 \\
PFANC3 & 1.14 & 27 \\
PFANC5 & 1.34 & 43 \\
PFANC7 & 1.29 & 42 \\
PFANC10 & 1.37 & 51 \\
PFANC15 & 1.45 & 59 \\
\hline
\end{tabular}

\subsection{Thermogravimetry $(T G)$}

TGA and DTG curves obtained in a nitrogen atmosphere for the virgin PFA and nanocomposites are shown in figure 4a and $b$, respectively. Mainly, it can be seen that the virgin PFA and all its nanocomposites exhibit one-step weight loss mechanism. ${ }^{32,33}$ The temperatures at which $10 \%$ and $60 \%$ weight loss occur and the residual weight rate as representative of thermal stability are listed in table 2. It is evident that the thermal stability of nanocomposites was improved compared to virgin polymer. Specifically, the thermal stability of nanocomposites that are prepared with small contents of clay (PFANC1, PFANC3 and PFANC5) increases with increasing of clay content; contrary to this, for relatively high clay contents (PFANC7, PFANC10 and PFANC15), it decreases with increasing of clay content; the PFANC5 presents the best thermal stability. These results show that the thermal stability of nanocomposites prepared is not related only to the clay content, but also to the state of existence of clay in the polymeric matrix (immiscibility, intercalation or exfoliation); therefore, it is related to the surface area between the polymeric matrix and the clay. Such results indicate that the introduction of clay might change the decomposition mechanism of PFA under high temperature. However, it is clear that these results support largely those obtained from RDX and MET.

\subsection{Mechanical properties}

Table 3 shows yield strength and tensile modulus of the virgin PFA and its nanocomposites (1, 3, 5, 7, 10 and $15 \mathrm{wt} \%)$. It is seen that the nanocomposites show improved mechanical properties compared with the virgin PFA; in other words the clay acted as a nucleating agent, increasing the stiffness of the material. It was also observed that there was an increase in tensile modulus (figure 5a) and yield strength (figure 5b) for the systems of PFA/MMT with the clay content; this increase in tensile modulus and yield strength is the maximum for PFANC15, approximately 79.01 and $555.55 \%$, respectively, in comparison with the virgin PFA. In addition, the state of the clay in the polymer matrix has a very important role in improving the mechanical properties of nanocomposites. For example, we note that the PFANC5 has better improved mechanical properties than those of PFANC7 despite the clay content of the latter. Therefore,

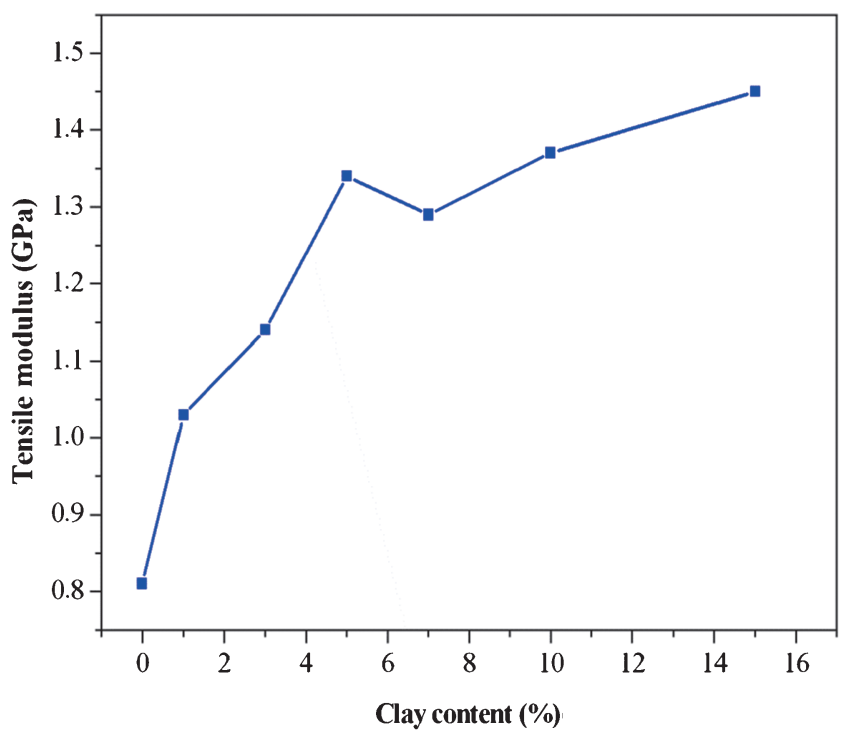

(a)

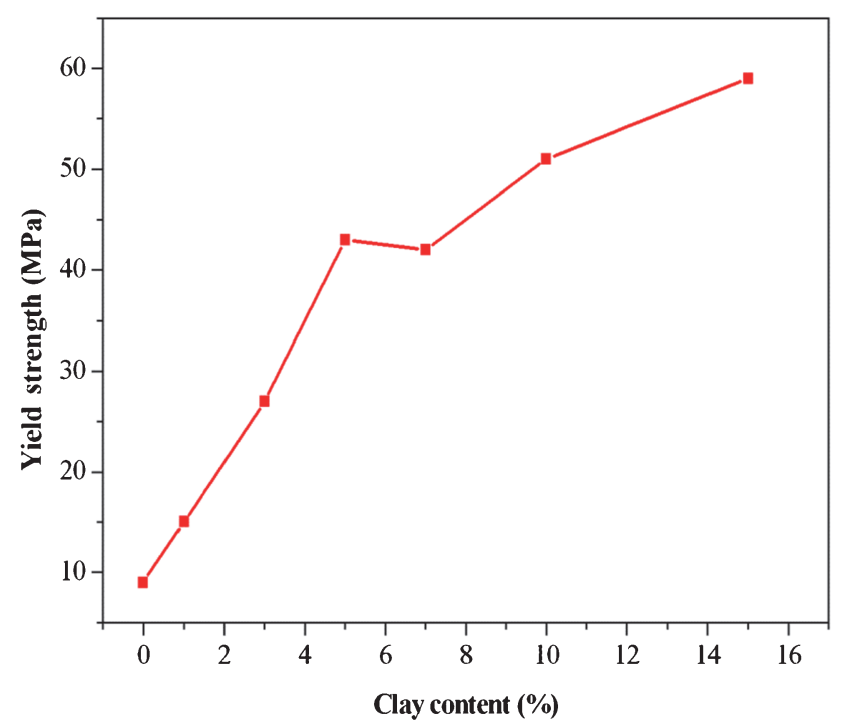

(b)

Figure 5. (a) Tensile modulus of poly(furfuryl alcohol) and its nanocomposites. (b) Yield strength of poly(furfuryl alcohol) and its nanocomposites.

as the nanocomposites are exfoliated, that the mechanical properties are more improved.

\section{Conclusion}

The effect of organoclay (12-MMT) prepared and used with different ratios on the properties of PFA/Algerian clay nanocomposites was studied. Infrared analysis confirmed that the PFA was polymerized. The results of XRD indicated that both nanocomposites prepared with 1, 3 and 5\% were exfoliated, but that prepared with $7 \%$ was intercalated and with $10 \%$ was partially intercalated among two basal planes of MMT, leading to an expansion of the interlayer spacing. Moreover, the nanocomposite prepared with $15 \%$ 
of clay is immiscible and almost no change of the interlayer spacing is observed. These results were confirmed by TEM. The results of thermogravimetry indicated that the nanocomposites showed largely higher thermal stability compared to pure polymer $\left(T<400^{\circ} \mathrm{C}\right)$. This is attributed to interactions between the set of polymer chains and the organic compounds of modified clays. The reinforcing effect of clay in the polymer was confirmed by the increased rigidity of the system. In general, the data showed 12-aminolauric acid to be efficient in the organophilization of clay and suggested that the Algerian clay can be used as a nanoparticle in PFA nanocomposites.

\section{References}

1. Kojima Y, Usuki A, Kawasumi M, Okada A, Kurauchi T and Kamigaito O 1993 J. Polym. Sci. Part A: Polym. Chem. 31983

2. Messersmith P B and Giannelis E P 1993 Chem. Mater. 51064

3. Giannelis E P 1996 Adv. Mater. 829

4. Krishnamoorti R, Vaia R A and Giannelis E P 1996 Chem. Mater. 817281

5. Gilman J W 1999 Appl. Clay Sci. 1531

6. Alexandre M and Dubois Ph 2000 Mater. Sci. Eng. 281

7. Biswas M and Ray S S 2000 Adv. Polym. Sci. 15167

8. Pinnavaia T J and Beall G W 2000 Polymer-clay nanocomposites (New York: Wiley Series in Polymer Science, Wiley)

9. Strawhecker K E and Manias E 2000 Chem. Mater. 122943

10. Ray S S and Okamoto M 2003 Prog. Polym. Sci. 281539

11. Lawrence P and Rina T 2008 Macromolecules 418682

12. Chuang I S and Maciel G E 1984 Macromolecules 171087

13. Choura M, Belgacem N M and Gandini A 1996 Macromolecules 293839

14. Principe M, Ortiz P and Martinez R 1999 Polym. Int. 48637
15. Gonzalez R, Figueroa J M and Gonzalez H 2002 Eur. Polym. J. 38287

16. Cesano F, Scarano D, Bertarione S, Bonino F, Damin A, Bordiga S et al 2008 J. Photochem. Photobiol. A: Chem. 196 143

17. Song C, Wang T and Qiu J 2009 Desalination 249486

18. Gandini A 2010 Polym. Chem. 1245

19. Belgacem M N and Gandini A 2008 Monomers, polymers and composites from renewable resources 1st ed. (Amsterdam: Elsevier Science) p. 115

20. Gandini A and Belgacem M N 1997 Prog. Polym. Sci. 221203

21. LeBaron P C, Wang Z and Pinnavaia T J 1999 Appl. Clay Sci. 1511

22. Belbachir M and Bensaoula A 2001 US Patent 2001/6,274,527 B1

23. Belbachir M and Bensaoula A 2003 US Patent 2003/0069446 A1

24. Akane O and Arimitsu U 1995 Mater. Sci. Eng. C3 109

25. Yahiaoui A, Belbachir M and Hachemaoui A 2003 Int. J. Mol. Sci. 4548

26. Kalpana S Katti, Debashis S, Dinesh R K, Pijush G and Devendra V 2006 Polymer 47403

27. Gonzalez R, Martinez R and Ortiz P 1992 Makromol. Chem. 1 193

28. Zhujian H, Pingxiao W, Hailing L, Wen L, Yajie Z and Nengwu Z 2014 RCS Adv. 46500

29. Kloprogge J T, Leisel H and Ray L F 2004 J. Raman Spectrosc. 35967

30. Farmer V C (ed) 1974 In Infrared spectra of minerals (London: Mineralogical Society) p. 331

31. Xue Q, Shen L T and Bai Y L 1995 Meas. Sci. Technol. 61557

32. Suda H and Haraya K 1997 J. Phys. Chem. B 1013988

33. Wang Z, Lu Z, Huang X, Xue R and Chen L 1998 Carbon 3651 